Editorial

\title{
Symmetry in Quantum Optics Models
}

\section{Lucas Lamata $\mathbb{D}$}

Departamento de Física Atómica, Molecular y Nuclear, Universidad de Sevilla, Apartado 1065, 41080 Sevilla, Spain; lucas.lamata@gmail.com

Received: 16 October 2019; Accepted: 17 October 2019; Published: 18 October 2019

This editorial introduces the successful invited submissions [1-5] to a Special Issue of Symmetry on the subject area of "Symmetry in Quantum Optics Models".

Quantum optics techniques can be regarded as the physical background of quantum technologies. These techniques are most often enhanced by symmetry considerations, which can simplify calculations as well as offer new insight into the models.

This Special Issue includes the novel techniques and tools for Quantum Optics Models and Symmetry, such as:

- Quasiprobability distribution functions employing fractional Fourier transforms [1].

- Ultrastrong coupling regime combined with parity symmetry for the nonclassical state of light generation [2].

- Employment of quantum optics models as the spin-boson system for simulating another quantum optics platform, as non-Markovian multi-photon Jaynes-Cummings models [3].

- $\quad$ Floquet topological techniques for analyzing optically driven semiconductors [4].

- Symmetries of the quantum Rabi model for its analysis in all possible parameter regimes [5].

Response to our call had the following statistics:

- $\quad$ Submissions (5);

- Publications (5);

- $\quad$ Article types: Research Article (5).

Authors' geographical distribution (published papers) is:

- China (2)

- $\quad$ Spain (2)

- $\quad$ Germany (2)

- $\operatorname{Mexico}(1)$

- UK (1)

- Chile (1)

- $\quad$ USA (1)

Published submissions are related to the aforementioned techniques and tools, and represent a selection of current topics in quantum optics models and their symmetries.

We found the edition and selections of papers for this book very inspiring and rewarding. We also thank the editorial staff and reviewers for their efforts and help during the process.

Conflicts of Interest: The author declares no conflict of interest. 


\section{References}

1. Anaya-Contreras, J.; Zúñiga-Segundo, A.; Moya-Cessa, H. Quasiprobability Distribution Functions from Fractional Fourier Transforms. Symmetry 2019, 11, 344. [CrossRef]

2. Cárdenas-López, F.; Romero, G.; Lamata, L.; Solano, E.; Retamal, J. Parity-Assisted Generation of Nonclassical States of Light in Circuit Quantum Electrodynamics. Symmetry 2019, 11, 372. [CrossRef]

3. Puebla, R.; Zicari, G.; Arrazola, I.; Solano, E.; Paternostro, M.; Casanova, J. Spin-Boson Model as A Simulator of Non-Markovian Multiphoton Jaynes-Cummings Models. Symmetry 2019, 11, 695. [CrossRef]

4. Lubatsch, A.; Frank, R. Behavior of Floquet Topological Quantum States in Optically Driven Semiconductors. Symmetry 2019, 11, 1246. [CrossRef]

5. Braak, D. Symmetries in the Quantum Rabi Model. Symmetry 2019, 11, 1259. [CrossRef]

C 2019 by the author. Licensee MDPI, Basel, Switzerland. This article is an open access article distributed under the terms and conditions of the Creative Commons Attribution (CC BY) license (http://creativecommons.org/licenses/by/4.0/). 